\title{
Analysis of elliptically polarized states in vertical-cavity-surface-emitting lasers
}

\author{
F. Prati* and P. Caccia \\ Dipartimento di Scienze, Università dell'Insubria, via Valleggio 11, I-22100 Como, Italy \\ M. Bache \\ Istituto Nazionale per la Fisica della Materia (INFM), Unità di Ricerca di Como, via Valleggio 11, I-22100 Como, Italy \\ F. Castelli \\ Dipartimento di Fisica, Università degli Studi di Milano, via Celoria 16, I-20133 Milano, Italy
}

(Received 22 September 2003; published 17 March 2004)

\begin{abstract}
We study the elliptically polarized states in the spin-flip model for vertical-cavity-surface-emitting lasers. The stability analysis reveals some unexpected features. In correspondence with particular values of the birefringence parameter, which are shown to scale very simply with the ratio of the spin-flip rate to the linewidth enhancement factor, the stability domain can be quite large. Moreover, in some cases two different dynamical regimes can arise from the destabilization of the elliptically polarized states, and they can coexist in a finite interval of the pump parameter. Finally, we show that the bifurcation from the lower frequency linearly polarized state to the elliptically polarized states can be subcritical when the linewidth enhancement factor is roughly smaller than 1 .
\end{abstract}

DOI: 10.1103/PhysRevA.69.033810

PACS number(s): 42.55.Px, 42.65.Sf, 42.25.Ja

\section{INTRODUCTION}

In the study of laser dynamics the electric field is usually treated as a scalar, in the sense that it is assumed to be always polarized along some fixed direction, and that the medium also responds in that direction. Yet, the limited validity of that assumption in some particular cases became clear since the early 1960 s, when the first He-Ne lasers displaying simultaneous oscillations of two orthogonally polarized modes appeared [1]. One of the first laser models where the vectorial nature of the electric field is taken into account is the so-called Zeeman laser [2]. Much more recently, the interest in vector lasers was renewed by the progress made especially with class- $B$ lasers [3].

VCSELs (vertical-cavity-surface-emitting lasers) are an example of class- $B$ lasers whose dynamics can be described adequately only using a vector model, because the two orthogonally polarized modes have almost equal gains. Polarization switching (PS) is commonly observed in VCSELs as one parameter, usually the injected current, is varied [4]. The frequencies of the two orthogonally polarized modes are split by birefringence. The switching from the high- to the lowfrequency mode is usually referred to as type I switching, and the opposite one as type II.

In the literature these phenomena have been attributed to two completely different mechanisms. In an earlier interpre-

\footnotetext{
*Also at Istituto Nazionale per la Fisica della Materia (INFM), Unità di Ricerca di Como, via Valleggio 11, I-22100 Como, Italy. Electronic address: franco.prati@uninsubria.it

†Electronic address: morten.bache@uninsubria.it

${ }^{\ddagger}$ Also at Istituto Nazionale per la Fisica della Materia (INFM), Unità di Ricerca di Milano, via Celoria 16, I-20133 Milano, Italy. Electronic address: fabrizio.castelli@mi.infn.it
}

tation, polarization switching is explained in terms of the modification of gain with increasing current due, for instance, to ohmic heating [5]. A different approach, the spinflip model (SFM), describes polarization switching as dynamical instabilities arising in a set of complex rate equations, where an essential role is played by the linewidth enhancement factor and the spin-flip rate [6].

A relevant difference between the two theoretical models is the following: SFM can account for both type I and type II switching, while only type I switching can be easily explained as an effect of the gain redshift with respect to the cavity modes due to carrier heating. Only if the photon energy dependence of cavity losses is included in the thermal model, type II switching can also appear, but it is always preceded by a type I switching [7].

Moreover, according to SFM, in type II switching the low-frequency polarized mode loses its stability with respect to the high-frequency mode when the frequencies of the two modes, split by birefringence in the cold cavity, lock due to nonlinear interactions with the semiconductor material. Under these conditions, the two-mode state which arises from the instability of the low-frequency mode is phase locked, which means that the laser emits elliptically polarized light. Increasing the injected current, the elliptically polarized state destabilizes and the VCSEL emits unpolarized light until it switches to the high-frequency mode. Thus, in SFM the elliptical polarized state plays an important role, in that it mediates the transition between the two orthogonally polarized states in type II switching.

That kind of switching has been recently demonstrated and carefully investigated [8,9]. The reported results look in agreement with SFM, but a complete comparison with the model requires, for what we said before, an accurate analysis of the properties of the elliptically polarized state in SFM, which is the aim of our paper.

To our knowledge the stability of the elliptically polarized 
state in the framework of the complete SFM has been studied only in a very limited number of cases, and mainly numerically $[10,11]$. A completely analytical study was performed within a two-equations model derived from SFM with asymptotic methods [12]. But one of the assumptions needed to derive that reduced model is that the birefringence parameter must be of the same order of magnitude as the recombination rate of carriers. Instead, as we will show in the following, the most interesting phenomena associated with the elliptically polarized state occur when the birefringence parameter is of the same order as the spin-flip rate, which is usually at least one order of magnitude larger than the recombination rate of carriers. Hence, there is no superposition between our results and those of Ref. [12].

In Sec. II we introduce the model equations and write the stationary equations for the elliptically polarized state. In Sec. III we present the results of the linear stability analysis of that state. The conditions under which the transition from the low-frequency linearly polarized state to the elliptically polarized state is subcritical, leading to bistability, are studied in Sec. IV, where the effects of noise in such a situation are also considered. Section $\mathrm{V}$ is devoted to the comments of the main results and the concluding remarks.

\section{MODEL AND ELLIPTICALLY POLARIZED SOLUTION}

According to the spin-flip model [6], the dynamical equations for the two circularly polarized components of the electric field $F_{ \pm}$, for the total carrier density $N$ and for the difference density $n$ of carriers with opposite spin are

$$
\begin{gathered}
\dot{F}_{ \pm}=k(1+i \alpha)(N \pm n-1) F_{ \pm}-\left(\gamma_{a}+i \gamma_{p}\right) F_{\mp}, \\
\dot{N}=\mu-N\left(1+\left|F_{+}\right|^{2}+\left|F_{-}\right|^{2}\right)-n\left(\left|F_{+}\right|^{2}-\left|F_{-}\right|^{2}\right), \\
\dot{n}=-n\left(\gamma_{s}+\left|F_{+}\right|^{2}+\left|F_{-}\right|^{2}\right)-N\left(\left|F_{+}\right|^{2}-\left|F_{-}\right|^{2}\right) .
\end{gathered}
$$

In these equations all variables are adimensional, and time has been scaled to the carrier lifetime, $k$ is the decay rate of photons in the cavity, $\gamma_{s}$ is the spin-flip rate, $\alpha$ is the linewidth enhancement factor, $\mu$ is the pump parameter, $\gamma_{a}$ and $\gamma_{p}$ are the linear anisotropy parameters, which describe, respectively, dichroism and birefringence.

We assume that linear anisotropies are aligned. As soon as this assumption is removed, it was shown in Ref. [13] that the orthogonal linearly polarized states are no longer solutions of SFM, and they are replaced by orthogonal elliptically polarized states, which reduce to the linearly polarized ones only when $\left|\gamma_{p}\right| \gg\left|\gamma_{a}\right|$. A similar competition between orthogonal elliptically polarized states was found in $\mathrm{Nd}$ doped fibre lasers [14]. Here we neglect these effects. The only possible stationary states of the VCSEL in our case are the two orthogonal linearly polarized states and the elliptical polarized states which arise from a stationary instability of the low-frequency linearly polarized state.

We remark also that, unlike in the models used for neodymium-doped fibre lasers [15] and Nd:YAG (YAG, yttrium aluminum garnet) lasers [16], in SFM stable twomode solutions, with the two orthogonal linearly polarized modes oscillating simultaneous at different frequencies, were never found. However, noise can sustain two-frequency emission in a VCSEL close to threshold and with small gain anisotropy [17].

We look for stationary solutions of the form $F_{ \pm}$ $=Q_{ \pm, s} \exp \left[i\left(\omega t \pm \psi_{s}\right)\right], N=1+\bar{N}_{s}, n=n_{s}$, where $Q_{ \pm, s}, \psi_{s}, \bar{N}_{s}$, and $n_{s}$ are independent of time, and $\omega$ is the frequency offset with respect to the reference frequency, which is the laser frequency in absence of any anisotropies. The linearly polarized solutions are well known, since they have been studied in detail in several previous works. We only remind that for these solutions

$$
I=2 Q_{ \pm, s}^{2}=\frac{\mu}{\mu_{\mathrm{th}}}-1, \quad \bar{N}_{s}=\mu_{\mathrm{th}}-1, \quad n_{s}=0,
$$

where $I$ is the stationary intensity, and the $x$-polarized and $y$-polarized solutions are characterized by the following thresholds, frequencies, and relative phases

$$
\begin{aligned}
& \mu_{\mathrm{th}, x}=1+\frac{\gamma_{a}}{k}, \quad \omega_{x}=-\gamma_{p}+\alpha \gamma_{a}, \quad \psi_{s, x}=0, \\
& \mu_{\mathrm{th}, y}=1-\frac{\gamma_{a}}{k}, \quad \omega_{y}=\gamma_{p}-\alpha \gamma_{a}, \quad \psi_{s, y}=\pi / 2 .
\end{aligned}
$$

Choosing for definiteness $\gamma_{p}$ positive, the above equations show that the $x$-polarized solution is the one oscillating at the lower frequency (the condition $\alpha\left|\gamma_{a}\right|<\gamma_{p}$ is usually verified), and it has the lower (higher) lasing threshold for $\gamma_{a}<0\left(\gamma_{a}>0\right)$.

The analysis of the elliptically polarized solution is a much more difficult task. In the framework of the complete SFM it has been performed previously only in the limit $\gamma_{a}$ $=0[10,11,18]$. The inclusion of dichroism makes the calculation very involved. We found it convenient to proceed as follows, although we cannot exclude that more convenient strategies exist. From the stationary equations for the carrier densities

$$
\begin{aligned}
& \mu=\left(\bar{N}_{s}+1\right)\left(1+Q_{+, s}^{2}+Q_{-, s}^{2}\right)+n_{s}\left(Q_{+, s}^{2}-Q_{-, s}^{2}\right), \\
& 0=\left(\bar{N}_{s}+1\right)\left(Q_{+, s}^{2}-Q_{-, s}^{2}\right)+n_{s}\left(\gamma_{s}+Q_{+, s}^{2}+Q_{-, s}^{2}\right),
\end{aligned}
$$

it is easy to obtain the stationary intensities $Q_{ \pm, s}^{2}$ in terms of the stationary densities $\bar{N}_{s}$ and $n_{s}$,

$$
Q_{ \pm, s}^{2}=\frac{\mu-\bar{N}_{s}-1 \mp \gamma_{s} n_{s}}{2\left(\bar{N}_{s}+1 \pm n_{s}\right)} .
$$

The stationary equations for the fields $F_{ \pm}$can be written as

$$
e^{\mp i 2 \psi_{s}}=\frac{\left[k(1+i \alpha)\left(\bar{N}_{s} \pm n_{s}\right)-i \omega\right]}{\gamma_{a}+i \gamma_{p}} \frac{Q_{ \pm, s}}{Q_{\mp, s}} .
$$

Multiplying side by side the above two equations we eliminate $Q_{ \pm, s}$ and $\psi_{s}$, and obtain a complex equation containing only $\bar{N}_{s}, n_{s}$, and $\omega$. Setting separately equal to zero the real 
and imaginary parts of this equation we get an expression for the oscillation frequency

$$
\omega_{\mathrm{el}}=\frac{k^{2} \alpha\left(\bar{N}_{s}^{2}-n_{s}^{2}\right)-\gamma_{a} \gamma_{p}}{k \bar{N}_{s}}
$$

and an auxiliary equation, biquadratic in both $\bar{N}_{s}$ and $n_{s}$,

$$
\begin{gathered}
\alpha^{2} k^{4} n_{s}^{4}+k^{2}\left[k^{2} \bar{N}_{s}^{2}\left(1-\alpha^{2}\right)+2 \alpha \gamma_{a} \gamma_{p}\right] n_{s}^{2}-k^{4} \bar{N}_{s}^{4}+k^{2} \bar{N}_{s}^{2}\left(\gamma_{a}^{2}\right. \\
\left.-\gamma_{p}^{2}\right)+\gamma_{a}^{2} \gamma_{p}^{2}=0,
\end{gathered}
$$

which will be used later. From Eq. (10) two couples of expressions for $\cos \left(2 \psi_{s}\right)$ and $\sin \left(2 \psi_{s}\right)$ can also be obtained, which read, taking into account Eq. (11),

$$
\begin{aligned}
& \cos \left(2 \psi_{s}\right)=\frac{Q_{ \pm, s}}{Q_{\mp, s}} \frac{k^{2}\left(n_{s} \pm \bar{N}_{s}\right)\left(\gamma_{p} n_{s} \alpha \pm \gamma_{a} \bar{N}_{s}\right)+\gamma_{a} \gamma_{p}^{2}}{k \bar{N}_{s}\left(\gamma_{a}^{2}+\gamma_{p}^{2}\right)}, \\
& \sin \left(2 \psi_{s}\right)=\frac{Q_{ \pm, s}}{Q_{\mp, s}} \frac{k^{2}\left(n_{s} \pm \bar{N}_{s}\right)\left(\gamma_{p} \bar{N}_{s} \mp \gamma_{a} n_{s} \alpha\right) \mp \gamma_{a}^{2} \gamma_{p}}{k \bar{N}_{s}\left(\gamma_{a}^{2}+\gamma_{p}^{2}\right)} .
\end{aligned}
$$

Of course these expressions are only apparently different, since they refer to the same stationary state. Imposing that the two expressions for $\cos \left(2 \psi_{s}\right)$ coincide and taking into account Eq. (9), we obtain an equation for $n_{s}^{2}$ in terms of only $\bar{N}_{s}$ and of the system parameters

$$
\begin{aligned}
n_{s}^{2}= & \frac{\bar{N}_{s}\left(\bar{N}_{s}+1\right)\left(\bar{N}_{s}+1-\mu\right) \alpha \gamma_{p}+\gamma_{a} \mathcal{A}\left(\bar{N}_{s}\right)}{\gamma_{a} \gamma_{s} \bar{N}_{s}+\alpha \gamma_{p}\left(\bar{N}_{s}+1-\mu-\gamma_{s}\right)} \\
\mathcal{A}\left(\bar{N}_{s}\right) \equiv & \bar{N}_{s}^{3} \gamma_{s}+\bar{N}_{s}^{2}\left(1+\gamma_{s}\right)+\bar{N}_{s}\left[\left(\gamma_{s}-1\right) \gamma_{p}^{2} / k^{2}-(\mu-1)\right] \\
& +\left(\mu+\gamma_{s}-1\right) \gamma_{p}^{2} / k^{2} .
\end{aligned}
$$

Finally, we may insert this equation into Eq. (12) to obtain a direct connection between $\mu$ and $\bar{N}_{s}$. We obtain a quadratic equation in $\mu$, which gives only one physical solution (the unphysical one simply gives values of $\mu$ below lasing threshold). In this way, for a given set of parameters $k, \alpha, \gamma_{s}, \gamma_{p}$, and $\gamma_{a}$ the elliptically polarized solutions as a function of the pump parameter $\mu$ can be constructed using $\bar{N}_{s}$ as a parameter. The following relations between $Q_{t, s}$ and the intensities $I_{x}$ and $I_{y}$ of the linearly polarized components of the field

$$
I_{x, y}=\frac{1}{2}\left[Q_{+}^{2}+Q_{-}^{2} \pm 2 Q_{+} Q_{-} \cos (2 \psi)\right],
$$

where the upper (lower) sign holds for the $x$-polarized $\left(y\right.$-polarized) solution, can be used to plot $I_{x}$ and $I_{y}$ as a function of $\mu$.

It is known that the elliptically polarized solution in the SFM model bifurcates from the $x$-polarized one. Therefore, the lower value of $\bar{N}_{s}$ for which the elliptically polarized solution can be found is that associated with the $x$-polarized solution, $\bar{N}_{s}=\gamma_{a} / k$. For this particular $\bar{N}_{s}$, the physical solution of the quadratic equation for $\mu$ coincides with the stability threshold of the $x$-polarized solution,

$$
\mu_{x, s}=\mu_{\mathrm{th}, x}\left[1+\frac{\gamma_{s}\left(\gamma_{p}^{2}+\gamma_{a}^{2}\right)}{\left(k \alpha-\gamma_{p}\right) \gamma_{p}+\left(k+\alpha \gamma_{p}\right) \gamma_{a}}\right] .
$$

The elliptically polarized solution begins to exist where the $x$-polarized solution ceases to be stable. In the following section we will investigate under which conditions the elliptically polarized solution is stable.

\section{LINEAR STABILITY ANALYSIS OF THE ELLIPTICALLY POLARIZED STATE}

The analysis of the stationary properties of the elliptically polarized state performed in the preceding section suggests that to study the stability of such a solution it is convenient to introduce, in place of the complex variables $F_{ \pm}$, the real variables $Q_{ \pm}, \chi$, and $\psi$ defined by

$$
F_{ \pm}=Q_{ \pm} e^{i(\chi \pm \psi)} .
$$

The quantities $Q_{ \pm}$and $2 \psi$ have the same meaning of real amplitudes and relative phase as the corresponding stationary quantities already encountered in the analysis of the stationary state; $2 \chi$ is the phase sum, and the derivative with respect to time of $\chi$ represents the (instantaneous) oscillation frequency with respect to the reference frequency. The important point is that the equation for $\chi$ is decoupled from the others, so we can limit our attention to the set of five equations:

$$
\dot{Q}_{+}=k(N+n-1) Q_{+}-\left[\gamma_{a} \cos (2 \psi)+\gamma_{p} \sin (2 \psi)\right] Q_{-},
$$

$$
\dot{Q}_{-}=k(N-n-1) Q_{-}-\left[\gamma_{a} \cos (2 \psi)-\gamma_{p} \sin (2 \psi)\right] Q_{+},
$$

$$
\begin{gathered}
\dot{\psi}=k \alpha n+\frac{\gamma_{a}}{2} \sin (2 \psi)\left(\frac{Q_{+}}{Q_{-}}+\frac{Q_{-}}{Q_{+}}\right)+\frac{\gamma_{p}}{2} \cos (2 \psi)\left(\frac{Q_{+}}{Q_{-}}-\frac{Q_{-}}{Q_{+}}\right), \\
\dot{N}=\mu-N\left(1+Q_{+}^{2}+Q_{-}^{2}\right)-n\left(Q_{+}^{2}-Q_{-}^{2}\right), \\
\dot{n}=-n\left(\gamma_{s}+Q_{+}^{2}+Q_{-}^{2}\right)-N\left(Q_{+}^{2}-Q_{-}^{2}\right) .
\end{gathered}
$$

However, the stability analysis remains a difficult task, because, unlike for the linear polarized states, here the set of equations obtained by linearizing Eqs. (20)-(24) around the stationary state are all coupled. Thus, the characteristic equation is a fifth degree equation of the form

$$
a_{5} \lambda^{5}+a_{4} \lambda^{4}+a_{3} \lambda^{3}+a_{2} \lambda^{2}+a_{1} \lambda+a_{0}=0,
$$

where, apart from $a_{5}=1$, the coefficients $a_{i}$ have extremely involved expressions, which we do not report here. Since we know that the instability of the elliptically polarized solution always gives rise to a dynamical regime, we focus on the 
condition for a Hopf instability which is, according to the Routh-Hurwitz criterion [19],

$$
\left(a_{1} a_{2}-a_{0} a_{3}\right)\left(a_{3} a_{4}-a_{2} a_{5}\right)-\left(a_{0} a_{5}-a_{1} a_{4}\right)^{2}<0 .
$$

The numerical study of this condition allowed us to obtain, for every value of the system parameters, the pump threshold $\mu_{\mathrm{el}}$ above which the elliptically polarized state becomes unstable. We fixed $k=600, \alpha=3$, and $\gamma_{s}=100$.

As for the amplitude anisotropy parameter $\gamma_{a}$, we consider two possible mechanisms that can contribute to it. First, the material strain, which we assume to be constant, neglecting a possible dependence on the injected current and then on the pump parameter $\mu$. Second, following the analysis developed in Ref. [20], we introduce in $\gamma_{a}$ a term proportional to the birefringence $\gamma_{p}$, which simulates the spectral dependence of gain, namely, the fact that the orthogonally polarized modes, being separated in frequency by an amount which is essentially $2 \gamma_{p}$, experience different gain. If the frequencies of the two modes lie to the left of the gain peak, the higher frequency state $(y)$ will have larger gain, hence the contribution to $\gamma_{a}$ is positive. In the opposite situation, the lower frequency state $(x)$ has the larger gain, and the contribution to $\gamma_{a}$ is negative. According to the above discussion we write $\gamma_{a}$ as

$$
\gamma_{a}=\gamma_{a, 0}+\eta \gamma_{p}
$$

where $\gamma_{a, 0}$ is the term related to dichroism and the proportionality coefficient $\eta$ is essentially the derivative of gain with respect to frequency. In our simulations we set $\gamma_{a, 0}$ $=0.1, \quad \eta=0.01$ when $\gamma_{a}$ is positive and $\gamma_{a, 0}=-0.1, \quad \eta$ $=-0.01$ when $\gamma_{a}$ is negative, with $\gamma_{p}$ always positive. The situation in which the two contributions have different signs has not been considered.

In Fig. 1 the threshold $\mu_{\mathrm{el}}$ for the elliptically polarized solution is plotted together with the instability thresholds of the linearly polarized solutions that we denote as $\mu_{x, s}, \mu_{x, H}$, and $\mu_{y, H}$. We distinguish between negative $\gamma_{a}$ [Fig. 1(a)] and positive $\gamma_{a}$ [Fig. 1(b)], because the instability thresholds $\mu_{x, H}$ and $\mu_{y, H}$ strongly depend on the sign of $\gamma_{a}$. Instead, $\mu_{\mathrm{el}}$ and $\mu_{x, s}$, are almost independent of it. The thresholds for the linearly polarized solutions are known from previous studies of the SFM. The expression for $\mu_{x, s}$ is given by Eq. (18); the other two thresholds have longer expressions that we do not report here. The main difference of Fig. 1 with respect to similar stability diagrams of SFM presented in several previous works is that the exact stability domain of the elliptically polarized solution is also shown. We will discuss later the properties of that stability domain. Before, we briefly resume the results about the stability of the linearly polarized solutions.

For negative $\gamma_{a}$ [Fig. 1(a)], the $x$-polarized solution is stable below the curve $\mu_{x, s}$, and the $y$-polarized solution is stable to the left of the curve $\mu_{y, H}$, which tends asymptotically to the vertical line $\gamma_{p}=\Gamma_{c}$, where the critical birefringence $\Gamma_{c}$ is defined as
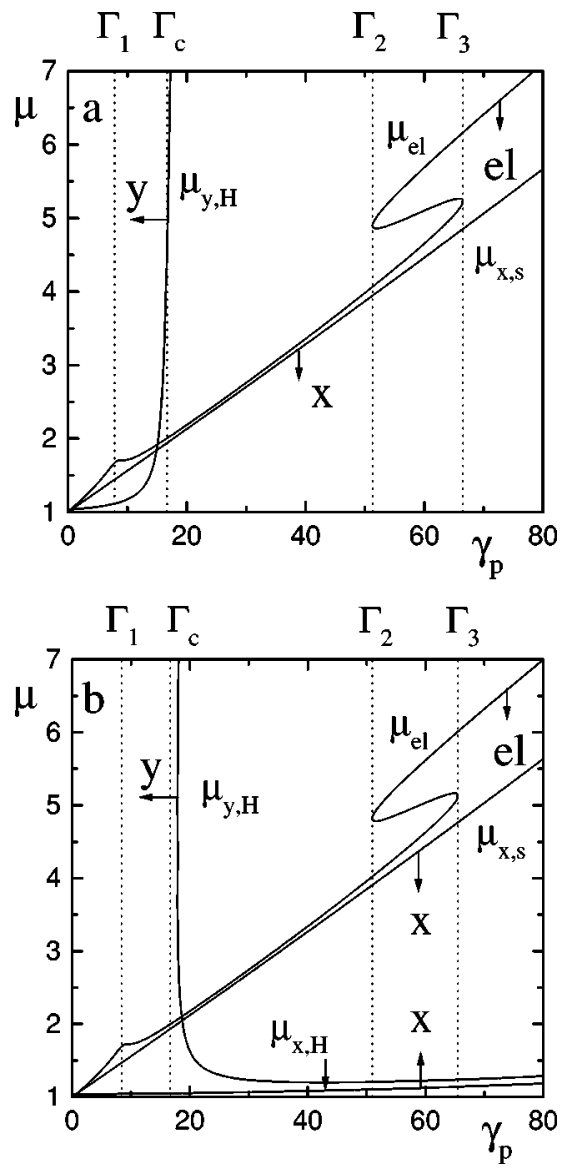

FIG. 1. Complete stability diagram for the linearly and elliptically polarized stationary solutions of SFM. The fixed parameters are $k=600, \gamma_{s}=100$, and $\alpha=3 . \gamma_{a}$ is negative in (a) and positive in (b), and it depends on $\gamma_{p}$ according to Eq. (27). The meaning of the instability thresholds $\mu_{\mathrm{el}}, \mu_{x, s}, \mu_{x, H}$, and $\mu_{y, H}$ and of the particular birefringence values $\Gamma_{c}, \Gamma_{1}, \Gamma_{2}$, and $\Gamma_{3}$ is explained in the text.

$$
\Gamma_{c}=\frac{\gamma_{s}}{2 \alpha} \text {. }
$$

For positive $\gamma_{a}$ [Fig. 1(b)], the $x$-polarized solution also experiences a Hopf instability close to threshold, and it is stable in the region between the curves $\mu_{x, H}$ and $\mu_{x, s}$; the $y$-polarized solution is stable to the left of the curve $\mu_{y, H}$, which has a $\mathrm{C}$ shape, extending from $\gamma_{p} \simeq \Gamma_{c}$ to the right. Actually, for reasonable values of the pump parameter $\mu$, only the lower branch of the $\mathrm{C}$ is visible. The upper branch can be seen for small values of $\mu$ only when gain saturation is considered [20].

Figures 1(a) and 1(b) allow to describe all the possible polarization switchings that may occur in the framework of SFM, and the role of the elliptically polarized state in these processes.

For negative $\gamma_{a}$ [Fig. 1(a)], at threshold the laser emits $x$-polarized light, because the $y$-polarized state is Hopf unstable. As the pump $\mu$ is increased, the laser switches to the elliptically polarized state at $\mu=\mu_{x, s}$ and to a dynamical state at $\mu=\mu_{\mathrm{el}}$. At this point, if $\gamma_{p}<\Gamma_{c}$, for a certain value of $\mu$ that cannot be predicted by the stability analysis, the laser 
will abandon the dynamical state and precipitate on the stable $y$-polarized solution, completing the PS II (from the lower to the higher frequency state). In contrast, for $\gamma_{p}>\Gamma_{c}$, the laser will remain on the dynamical state for any $\mu>\mu_{\mathrm{el}}$.

For positive $\gamma_{a}>0$ [Fig. 1(b)], the $x$-polarized state is Hopf unstable close to the lasing threshold, and the first emitted mode is always the $y$-polarized one. If $\gamma_{p}<\Gamma_{c}$ the laser will remain always in the $y$-polarized state increasing the pump $\mu$ from the lasing threshold. If $\gamma_{p}>\Gamma_{c}$ a PS I switching to the $x$-polarized state (from the higher to the lower frequency state) occurs at $\mu=\mu_{y, H}$. Then, the laser switches to the elliptically polarized solution at $\mu=\mu_{x, s}$ and finally it enters the dynamical regime at $\mu=\mu_{\mathrm{el}}$ and it remains there for any $\mu$.

The transition to the elliptically polarized state is associated with a PS II when the two conditions $\gamma_{a}<0$ and $\gamma_{p}<\Gamma_{c}$ hold. The transition is not followed by a PS II, and it is preceded by a PS I switching, for $\gamma_{a}>0$ and $\gamma_{p}>\Gamma_{c}$.

Let us analyze now the stability domain of the elliptically polarized solution, which displays some unexpected features.

First, the instability threshold $\mu_{\mathrm{el}}$ always presents a maximum at a characteristic value of the birefringence $\Gamma_{1}$, smaller than $\Gamma_{c}$. Around this point the size of the stable domain sharply increases.

Second, the stability domain displays an S-shaped region, between the birefringence values $\Gamma_{2}$ and $\Gamma_{3}$. The existence of this region implies that for $\Gamma_{2}<\gamma_{p}<\Gamma_{3}$, as the pump $\mu$ increases, the laser crosses three stability boundaries $\mu_{\mathrm{el}, i}, i$ $=1,2,3$. In other words, in this region there are two disjoint stability domains for the elliptically polarized solution $\mu_{x, s}<\mu<\mu_{\mathrm{el}, 1}$ and $\mu_{\mathrm{el}, 2}<\mu<\mu_{\mathrm{el}, 3}$.

Since these domains are always placed to the right of $\Gamma_{c}$, i.e., in a region where, above $\mu_{x, s}$, both linearly polarized solutions are unstable, as soon as the first stability boundary $\mu_{\mathrm{el}, 1}$ is crossed in a forward sweep of the pump parameter $\mu$ [Fig. 2(a)], the laser enters a dynamical regime, and it remains there even when $\mu$ is further increased and the upper stability domain is reached.

But our numerical study of the dynamical equations (1)-(3) revealed an interesting feature: for a certain value $\bar{\mu}>\mu_{\mathrm{el}, 3}$ of the pump, the laser switches to a new dynamical regime, characterized by oscillations with different frequency and amplitudes. For the chosen parameters, the transition occurs at about $\bar{\mu}=6.8$, and this value represents a true deterministic threshold, in that it is independent from the initial seed of the noise generator.

If now the pump is decreased in a backward sweep [Fig. 2(b)], we observe that, unlike in the forward sweep, the upper stability domain of the elliptically polarized solution becomes accessible, and stable elliptically polarized light is emitted between the pump values $\mu_{\mathrm{el}, 2}$ and $\mu_{\mathrm{el}, 3}$. Only below $\mu_{\mathrm{el}, 2}$ the same behavior as in the forward sweep is found. But this hysteretic behavior is not observed, and the backward sweep is always identical to the forward one, if the backward sweep of the pump parameter $\mu$ begins when the laser is still in the first dynamical state, i.e., below $\bar{\mu}$.

We can conclude that, among the three Hopf bifurcations of the elliptically polarized state that exist for $\Gamma_{2}<\gamma_{p}<\Gamma_{3}$, two $\left(\mu_{\mathrm{el}, 1}\right.$ and $\left.\mu_{\mathrm{el}, 3}\right)$ are supercritical, while the other one $\left(\mu_{\mathrm{el}, 2}\right)$ is subcritical. The two observed dynamical states are
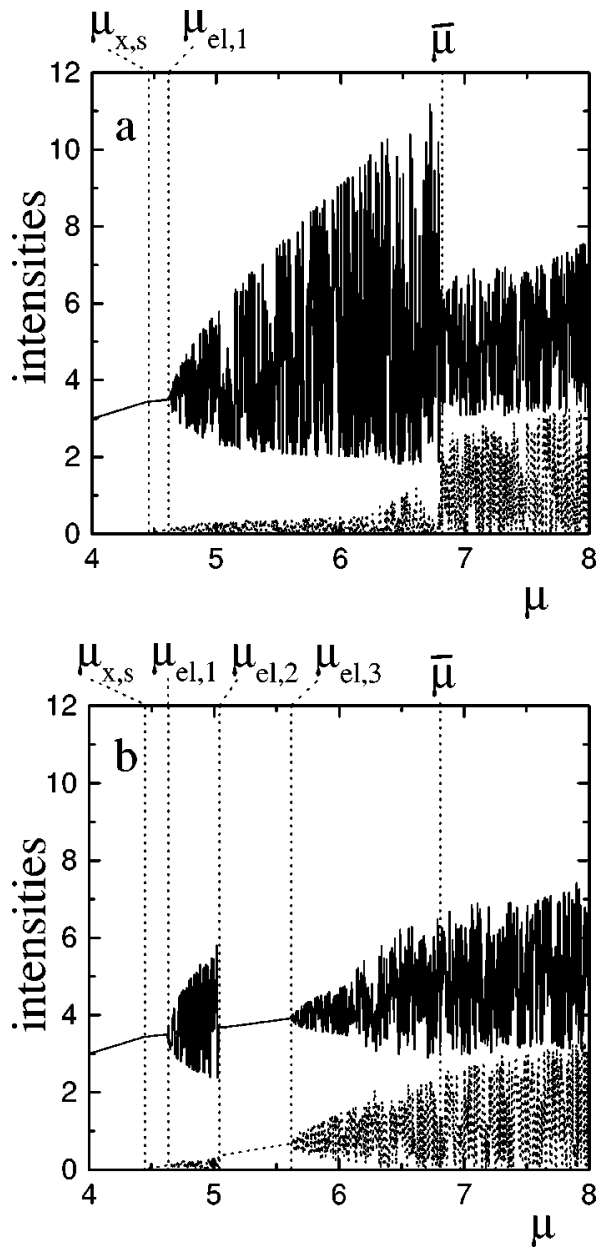

FIG. 2. Behavior of the intensity of the $x$-polarized (solid line) and $y$-polarized (dashed line) emitted light during a forward (a) and backward (b) scan of the pump parameter $\mu$. The parameters are the same as in Fig. 1(b), with $\gamma_{p}=60$. The pump parameter $\mu$ is adiabatically varied at a rate of one unit every $0.1 \mathrm{~ms}$. The meaning of the particular values $\mu_{x, s}, \mu_{e l, 1}, \mu_{e l, 1}, \mu_{e l, 1}$, and $\bar{\mu}$ of the pump parameter $\mu$ is explained in the text.

associated with the two supercritical Hopf bifurcations and they coexist in a finite interval of values between $\mu_{\mathrm{el}, 3}$ and $\bar{\mu}$. Instead, between $\mu_{\mathrm{el}, 2}$ and $\mu_{\mathrm{el}, 3}$, i.e., in the upper stability domain, bistability between the elliptically polarized state and the first dynamical regime is observed.

Another rather trivial consequence of the S-shaped stability domain is that, in a forward sweep of the pump parameter $\mu$, the size of the stability domain for the elliptically polarized solution sharply varies in correspondence with $\Gamma_{3}$, being much larger to the right of this birefringence value.

A better estimate of the relative importance of the elliptically polarized states in a VCSEL can be provided by the quantity

$$
r=\frac{\mu_{\mathrm{el}}-\mu_{x, s}}{\mu_{x, s}-\mu_{\mathrm{th}, x}},
$$

which measures the relative size of the stability domains of the elliptically polarized state to the $x$-polarized state. We plot in Fig. 3 this quantity for the same parameters as in Fig. 


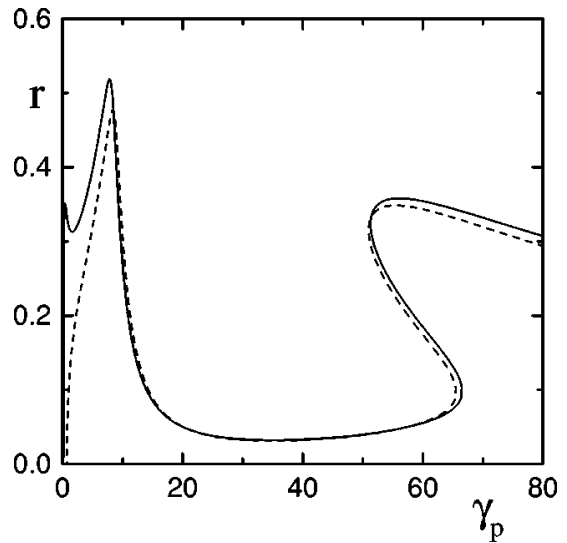

FIG. 3. Plot of the ratio $r$ which measures the relative size of the stability domain for the elliptically polarized solution. Same parameters as in Fig. 1. Solid line for negative $\gamma_{a}$ and dashed line for positive $\gamma_{a}$.

1. This figure shows that the ratio $r$ reaches comparable values in correspondence with the two critical parameters $\Gamma_{1}$ and $\Gamma_{2}$. In these points the size of the stability domain of the elliptically polarized state could be as large as $30 \%$ to $50 \%$ of the stability domain of the $x$-polarized state, while there is a large region between $\Gamma_{c}$ and $\Gamma_{2}$ where it is only a few percent.

Our calculations showed that the shape of the stability domain of the elliptically polarized solution depends strongly on the spin-flip rate $\gamma_{s}$. In Fig. 4 we consider $\gamma_{a}=0$ and compare the curves for $r$ obtained with $\gamma_{s}=50,100,150$. Clearly the critical values $\Gamma_{1}, \Gamma_{2}$, and $\Gamma_{3}$ increase as $\gamma_{s}$ increases. In particular, we checked, using also other values for $\alpha$, that $\Gamma_{1}$ and $\Gamma_{2}$ are approximated very well by the simple equations

$$
\begin{gathered}
\Gamma_{1} \simeq \frac{\gamma_{s}}{4 \alpha}=\frac{\Gamma_{c}}{2}, \\
\Gamma_{2} \simeq \frac{3 \gamma_{s}}{2 \alpha}=3 \Gamma_{c} .
\end{gathered}
$$

The ratio $\gamma_{s} / \alpha$, which contains the main ingredients of SFM, i.e., spin flip and saturable dispersion, reveals in this way its

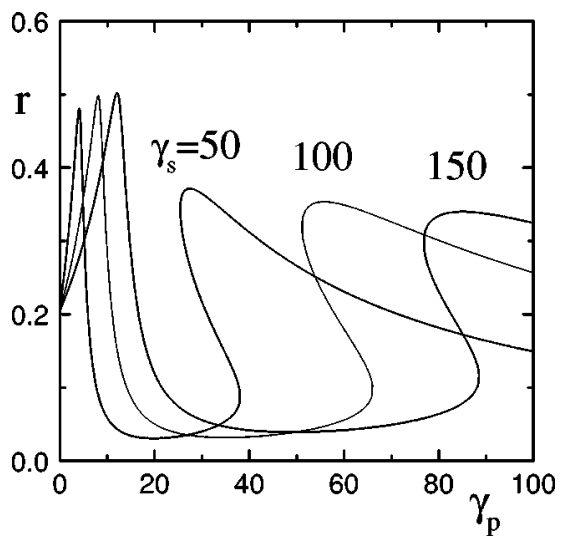

FIG. 4. Plot showing the ratio $r$ for the indicated values of the spin-flip rate $\gamma_{s}$. The other parameters are the same as in Fig. 1, with $\gamma_{a}=0$. ubiquitous character in SFM. It determines not only the position of the Hopf instability domain for the $y$-polarized solution, as seen previously, but also the shape of the stability domain for the elliptically polarized solution.

\section{SUBCRITICAL BIFURCATION TO THE ELLIPTICALLY POLARIZED STATE}

In this section we want to analyze if and under which conditions the static bifurcation which leads to the elliptically polarized state from the $x$-polarized state may be subcritical. If this happens, the elliptically polarized solution which emerges from the $x$-polarized state at the bifurcation point $\mu_{x, s}$ will extend initially to the left of $\mu_{x, s}$, until a turning point $\mu_{\text {sub }}$ is reached.

The interest for such a situation lies on the fact that it implies that both the elliptically polarized state and the $x$-polarized state exist in the interval $\mu_{\text {sub }}<\mu<\mu_{x, s}$. Therefore, if the elliptically polarized state is stable in that interval there is bistability between the two states. In a deterministic system, a hysteresis cycle would be observed sweeping forward and backward the pump parameter $\mu$, with discontinuous jumps from one state to the other at $\mu=\mu_{x, s}$ and $\mu$ $=\mu_{\text {sub }}$.

The analysis of the possible subcritical character of the bifurcation can be carried out much more simply in the limit $\gamma_{a}=0$. Therefore, we will consider first that limit and then we will extend the result to the general situation. For $\gamma_{a}=0$ we know that $\bar{N}_{s}=0$ in the $x$-polarized state and $\bar{N}_{s}>0$ in the elliptically polarized state. To investigate the condition for a subcritical bifurcation we consider the equation that relates $\bar{N}_{s}$ with $\mu$, which, in this limit, reduces to [11]

$$
\begin{aligned}
& k^{2}\left[\bar{N}_{s}\left(\gamma_{s}+\mu-\bar{N}_{s}-1\right)+\alpha^{2}\left(\bar{N}_{s}+1\right)\left(\mu-\bar{N}_{s}-1\right)\right] \\
& \quad \times\left[\bar{N}_{s}\left(\gamma_{s}+1\right)+1-\mu\right]+\gamma_{p}^{2}\left(\gamma_{s}+\mu-\bar{N}_{s}-1\right)^{2}=0 .
\end{aligned}
$$

We assume that $\bar{N}_{s}$ is positive and much smaller than 1 . This allows for a series expansion of $\mu$ of the type $\mu=\mu_{x, s}+p \bar{N}_{s}$ $+O\left(\bar{N}_{s}^{2}\right)$. The bifurcation is subcritical (supercritical) for negative (positive) $p$. The calculated coefficient $p$ is

$$
p=1-\frac{k \gamma_{s}\left(k-k \alpha^{2}+2 \alpha \gamma_{p}\right)}{2\left(k \alpha-\gamma_{p}\right)^{2}},
$$

and the bifurcation is subcritical when the inequality

$$
2\left(k \alpha-\gamma_{p}\right)^{2}<k \gamma_{s}\left(k-k \alpha^{2}+2 \alpha \gamma_{p}\right)
$$

is satisfied. In terms of the parameter $\alpha$ this condition can be reformulated as

$$
\frac{\gamma_{p}}{k}-\frac{1}{k} \sqrt{\frac{\gamma_{s}\left(k^{2}+\gamma_{p}^{2}\right)}{2+\gamma_{s}}}<\alpha<\frac{\gamma_{p}}{k}+\frac{1}{k} \sqrt{\frac{\gamma_{s}\left(k^{2}+\gamma_{p}^{2}\right)}{2+\gamma_{s}}}
$$

Taking into account that $\alpha$ is positive and $\gamma_{s} \gg 1$ we can consider the single approximated condition 


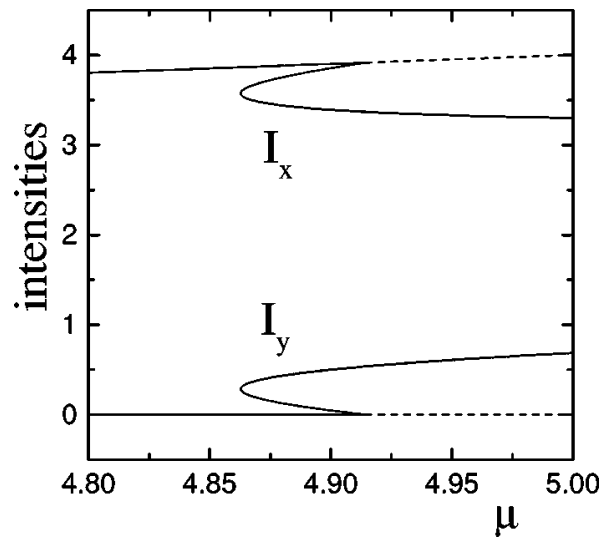

FIG. 5. Stationary intensities for the two orthogonally polarized components of the electric field in a region around the instability threshold of the $x$-polarized component for $\kappa=600, \gamma_{s}=100, \alpha$ $=0.9, \gamma_{p}=20$, and negative $\gamma_{a}$. The $x$-polarized state bifurcates subcritically to the elliptically polarized state.

$$
\alpha<\frac{\gamma_{p}}{k}+\sqrt{1+\left(\frac{\gamma_{p}}{k}\right)^{2}} .
$$

Since we have also $\gamma_{p} \ll k$ for realistic values of the birefringence parameter $\gamma_{p}$, it turns out that the condition for a subcritical bifurcation is simply

$$
\alpha<1+\frac{\gamma_{p}}{k}+O\left(\frac{\gamma_{p}}{k}\right)^{2}
$$

If the effects of dichroism are also included allowing $\gamma_{a}$ for being different from zero, we found that the critical value for the $\alpha$ parameter can be approximated as

$$
\alpha \simeq \alpha_{c}=1+\frac{\gamma_{p}}{k}-2 \frac{\gamma_{a}}{\gamma_{p}}
$$

We see that $\alpha_{c}$ is larger for $\gamma_{a}<0$, which is also the condition under which the $x$-polarized solution is the first lasing

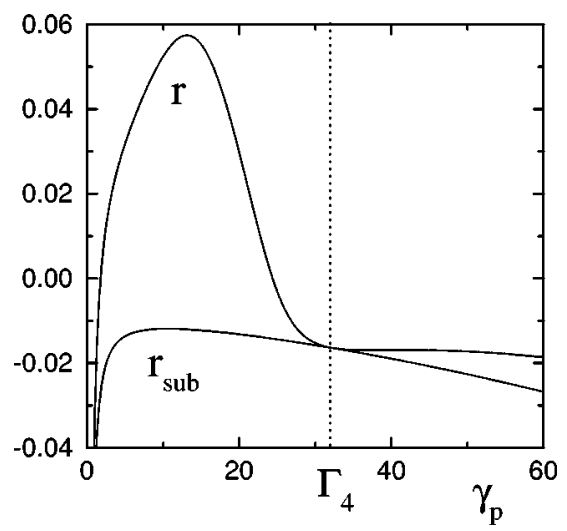

FIG. 6. The ratio $r_{\text {sub }}$, whose absolute value measures the relative size of the region where, for a subcritical bifurcation, the elliptically polarized and $x$-polarized linearly polarized solutions coexist, is shown together with $r$. Same parameters as in Fig. 5, apart from $\gamma_{p}$ which is now a free parameter. At $\gamma_{p}=\Gamma_{4}$, the elliptically polarized solution is always unstable. solution and hence the bifurcation to the elliptically polarized state can be more easily observed.

In Fig. 5 the stationary intensities of the elliptically polarized state are shown in one case where the bifurcation from the $x$-polarized state is subcritical, for $k=600, \gamma_{s}=100, \alpha$ $=0.9, \gamma_{p}=20$, and $\gamma_{a}=-0.3\left(\gamma_{a, 0}=-0.1, \eta=-0.01\right)$. The linearly polarized state is also shown, with dashed lines above the bifurcation point $\mu_{x, s}$, where it is unstable. For this choice of the parameters Eq. (38) gives the critical value $\alpha_{c}$ $=1.063$. We checked numerically that the exact value is 1.054

Similarly to what we have done in the preceding section, we introduce the ratio

$$
r_{\mathrm{sub}}=\frac{\mu_{\mathrm{sub}}-\mu_{x, s}}{\mu_{x, s}-\mu_{\mathrm{th}, x}}
$$

which is negative by definition, and whose absolute value is the relative size of the region where the elliptically and $x$-polarized solution coexist with respect to the region where the latter is stable.

In Fig. 6 we show both $r$ and $r_{\text {sub }}$ as a function of $\gamma_{p}$ for the same parameters as in Fig. 5. As in the previous cases, $r$ presents a maximum for a small value of the birefringence which, however, is different from $\gamma_{s} / 4 \alpha$. In the coexistence region there is bistability until $r$ remains positive. For negative $r$ the elliptically polarized state is stable only in a part of the coexistence region. In our calculation we found that there is always a fourth critical value $\Gamma_{4}$ of the birefringence parameter at which the stability domain of the elliptically polarized solution shrinks to zero $\left(\mu_{\mathrm{el}}=\mu_{\mathrm{sub}}\right)$, and the whole upper branch is unstable. We checked that also $\Gamma_{4}$ increases with increasing $\gamma_{s}$, but we were not able to determine a simple link with $\Gamma_{c}$, also because the condition $\alpha<\alpha_{c}$ for the subcritical bifurcation does not allow to explore a wide range of values for $\alpha$.

In a real experiment the deterministic hysteresis cycle between $\mu_{\text {sub }}$ and $\mu_{x, s}$ will be affected by the presence of noise. If noise is small, this simply means that the size of the cycle is reduced. Larger noise induces irregular jumps between the two coexisting states for any value of $\mu$, and the hysteresis cycle could disappear. In this case the existence of an underlying bistability can be inferred looking at the intensity histograms of the two linearly polarized modes, which in a certain interval of injected current should present two distinct peaks. Similar effects were observed in presence of bistability between orthogonally polarized first-order transverse modes [21].

In order to simulate this behavior we introduced in Eqs. (1) some sources of Gaussian noise $\xi_{ \pm}$which reproduce the effects of spontaneous emission. The complex functions $\xi_{ \pm}$ have a zero mean value and are $\delta$ correlated in time

$$
\begin{gathered}
\left\langle\xi_{ \pm}(t)\right\rangle=0, \\
\left\langle\xi_{ \pm}(t) \xi_{ \pm}\left(t^{\prime}\right)\right\rangle=0,
\end{gathered}
$$



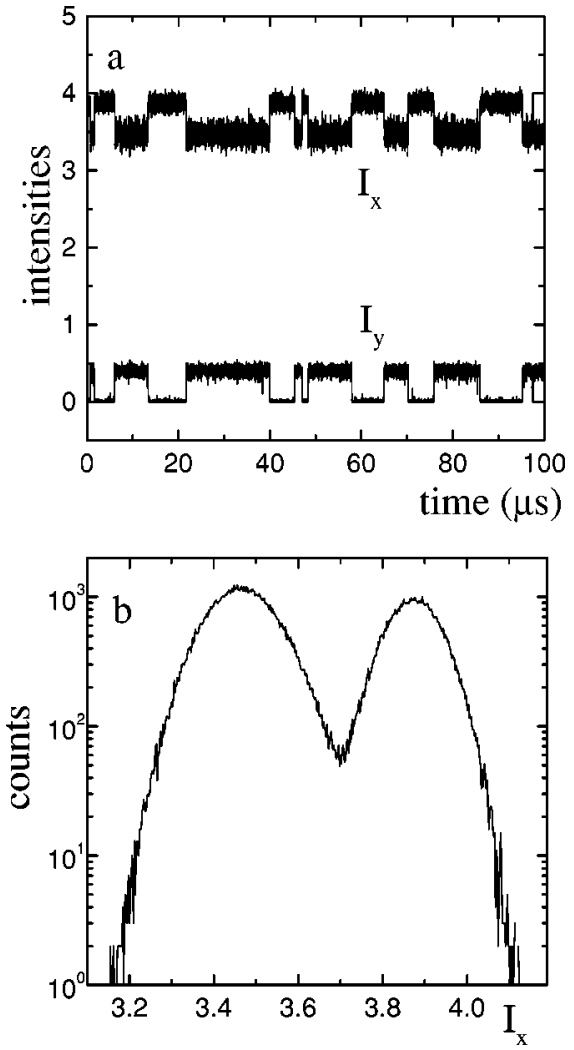

FIG. 7. Dynamics of the electric field in a bistable case, where noise induces jumps from one state to the other. Same parameters as in Fig. 5, with $\mu=4.878$. Time evolution of the intensities of the two orthogonally polarized components of the field (a), and histogram for the $x$-polarized component, showing a two-peak distribution (b).

$$
\left\langle\xi_{ \pm}^{*}(t) \xi_{ \pm}\left(t^{\prime}\right)\right\rangle=\beta \delta_{+,+} \delta_{-,-} \delta\left(t-t^{\prime}\right) .
$$

The strength of noise is measured by the parameter $\beta$. For $\beta=2.5 \times 10^{-3}$ we still observe a hysteresis cycle, although its size is much smaller than that predicted by the deterministic equations. For $\beta=5 \times 10^{-3}$ the hysteresis cycle disappears, and two-peak histograms are found. Figure 7(a) shows the time evolution of the two mode intensities on a time interval of $100 \mu$ s for the same parameters as in Fig. 5 and $\mu=4.878$. Irregular jumps between the linearly polarized state $I_{x}=3.881, I_{y}=0$ and the elliptically polarized state $I_{x}=3.447, I_{y}=0.424$ are clearly visible. The twopeak histogram of Fig. 7(b) has been obtained recording $2 \times 10^{5}$ data, with a sampling rate of 2 Gsamples/s, while the time step in the numerical integration was $10 \mathrm{ps}$.

With the chosen value of $\beta$ two-peak histograms were found for $4.876 \leqslant \mu \leqslant 4.882$, i.e., in an interval of length 0.006 scaled to the threshold pump, which in the theoretical model is unity. This interval looks very small, but we remark that in Refs. [8,9] two-peak histograms in correspondence with a PS II were reported in an interval $\Delta I=30 \mu \mathrm{A}$, when the lasing threshold was $I_{\text {th }}=4.3 \mathrm{~mA}$, which means $\Delta I / I_{\text {th }}$ $\simeq 0.007$. However, the analogy between those experiments and our numerical simulation probably ends here, because in the experiments the two states between which the VCSEL jumps are dynamical states, rather than elliptically and linearly polarized stationary states.

\section{CONCLUSIONS}

In this paper we have studied the stability of the elliptically polarized solutions in the SFM. The importance of this analysis is due to the fact that, according to the SFM, elliptically polarized states always exist as soon as the lowerfrequency linearly polarized state destabilizes. Hence, these states play an important role in the so-called type II polarization switching, from the lower- to the high-frequency mode. In spite of this, the elliptically polarized states have been often considered of little importance, because it was assumed that they are stable only in a very limited range of the pump parameter.

Our analysis showed that this is not always the case, and in correspondence with particular values of the birefringence parameter the size of the stability domain of the elliptically polarized states can be considerably large.

Quite interestingly, those particular values of the birefringence are shown to scale rationally with the ratio $\gamma_{s} / \alpha$, which was already known to determine the position of the Hopf instability domain for the high-frequency linearly polarized state. Thus, the role of the ratio of the spin-flip rate to the linewidth enhancement factor in the SFM is further emphasized.

The linear stability analysis revealed also that in some cases there is an interval of values for the birefringence parameter for which, as the pump parameter is increased, two disjoint stability domains for the elliptically polarized states are met. The dynamical simulations showed that, in correspondence with the two supercritical Hopf bifurcations associated with the upper stability boundaries of the two domains, two different dynamical regimes are created, and they can coexist in a finite interval of the pump parameter.

We have also shown that when the parameter $\alpha$ is roughly smaller than 1, the bifurcation from the lower-frequency linearly polarized state to the elliptically polarized state can be subcritical. In that case there is bistability between the two states and a hysteresis cycle can be observed sweeping forward and backward the pump parameter. In presence of noise, however, the hysteresis can disappear, and there is an interval of values for the pump parameter where two-peak histograms in the intensity of the two perpendicular components of the emitted light can be observed, due to noise induced jumps from one state to the other. Values of $\alpha$ smaller than 1 are not common, but they can be achieved by temperature tuning the laser frequency to the blue side of the gain spectrum [22,23], which is also the condition for which the lower-frequency state is stable at threshold, and the bifurcation to the elliptical state can occur quite close to threshold.

\section{ACKNOWLEDGMENT}

This work was funded by the European Commission through the network VISTA (VCSELs for Information Society Technology Applications, Contract No. HPRN-CT-200000034). 
[1] W. Culshaw and J. Kannelaud, Phys. Rev. 145, 257 (1966).

[2] M. Sargent III, M. O. Scully, and W. E. Lamb, Jr., Laser Physics (Westview Press, Boulder, CO, 1974).

[3] Yu. V. Loiko, A. M. Kul'minskii, and A. P. Voitovich, Opt. Commun. 210, 121 (2002).

[4] C. J. Chang-Hasnain, J. P. Harbison, G. Hasnain, A. Von Lehmen, L. T. Florez, and N. G. Stoffel, IEEE J. Quantum Electron. 27, 1402 (1991).

[5] K. D. Choquette, D. A. Richie, and R. E. Leibenguth, Appl. Phys. Lett. 64, 2062 (1994).

[6] M. San Miguel, in Semiconductor Quantum Optoelectronics, edited by A. Miller, M. Ebrahimzadeh, and D. M. Finlayson (Institute of Physics Publishing, Bristol, 1999), p. 339.

[7] B. Ryvkin, K. Panajotov, A. Georgievski, J. Danckaert, M. Peeters, G. Verschaffelt, H. Thienpont, and I. Veretennicoff, J. Opt. Soc. Am. B 16, 2106 (1999).

[8] T. Ackemann and M. Sondermann, Appl. Phys. Lett. 78, 3574 (2001).

[9] T. Ackemann and M. Sondermann, Proc. SPIE 4286, 44 (2001).

[10] M. Travagnin, M. P. van Exter, A. K. Jansen van Doorn, and J. P. Woerdman, Phys. Rev. A 54, 1647 (1996).

[11] J. Martin-Regalado, F. Prati, M. San Miguel, and N. B. Abra- ham, IEEE J. Quantum Electron. 33, 765 (1997).

[12] T. Erneux, J. Danckaert, K. Panajotov, and I. Veretennicoff, Phys. Rev. A 59, 4660 (1999).

[13] M. Travagnin, Phys. Rev. A 56, 4094 (1997).

[14] P. Khandokhin, Ya. Khanin, Yu. Mamaev, N. Milovsky, E. Shirokov, S. Bielawski, D. Derozier, and P. Glorieux, Quantum Semiclassic. Opt. 10, 97 (1998).

[15] H. Zeghlache and A. Boulnois, Phys. Rev. A 52, 4229 (1995).

[16] M. Brunel, O. Emile, M. Alouini, A. Le Floch, and F. Bretenaker, Phys. Rev. A 59, 831 (1999).

[17] M. Sondermann, M. Weinkath, T. Ackemann, J. Mulet, and S. Balle, Phys. Rev. A 68, 033822 (2003).

[18] M. Travagnin, M. P. van Exter, and J. P. Woerdman, Phys. Rev. A 56, 1497 (1997).

[19] H. Haken, Synergetics-An Introduction (Springer, Berlin, 1974).

[20] F. Prati, P. Caccia, and F. Castelli, Phys. Rev. A 66, 063811 (2002).

[21] F. Prati, G. Giacomelli, and F. Marin, Phys. Rev. A 62, 033810 (2000)

[22] X. Wang, H. Li, J. McInerney, and J. Malloy (unpublished).

[23] H. D. Summers, P. Dowd, I. H. White, and M. R.T. Tan, IEEE Photonics Technol. Lett. 7, 736 (1995). 